Original Articles

\title{
QT Dispersion is Increased in Diabetic Patients with Foot Ulcer
}

\author{
Eiichi Imano1, Rieko Yoshioka², Yoshihisa Nakatani', Katsumi Arai', Masaaki \\ Motomura', Tsutomu Kanda', Yoshimitsu Yamasaki', and Masatsugu Hori²
}

'Department of Gastroenterology and Metabolic Disease, Osaka Prefectural General Hospital, Osaka, Japan.

${ }^{2}$ First Department of Medicine, Osaka University School of Medicine, Osaka, Japan.

\begin{abstract}
QT dispersion, a measure of inhomogenous ventricular repolarization, was measured in diabetic patients with foot ulcer. We recruited 75 patients with non-insulin-dependent diabetes mellitus: patients with neuropathic ulcer $(n=15, \mathrm{NU}$ group), with ischemic ulcer ( $n=20$, IU group), with previous myocardial infarction $(n=20$, MI group) and without any diabetic microangiopathies ( $n=20$, DC group). We also studied normal control subjects ( $n=15$, NC group). The interlead variability of rate-corrected QT interval (QTC dispersion) was calculated. QTc interval in the MI group was significantly higher than that in the NC or DC but showed no difference in the NU and IU groups. QTC dispersion in the IU (54 \pm 15 $\mathrm{msec})$ as well as $\mathrm{MI}(60 \pm 21 \mathrm{msec})$ group were significantly higher than the NC $(36 \pm 18$ $\mathrm{msec})$ or DC group $(39 \pm 14 \mathrm{msec})$. This may be due to complicated coronary artery disease in the IU group. Furthermore, QTC dispersion was also increased (49 $\pm 14 \mathrm{msec})$ in the NU group in which cardiac autonomic nervous dysfunction was suggested. Patients with both types of diabetic ulcer demonstrated increased QT dispersion due to atherosclerosis or neurological disorder. J Atheroscler Thromb, $2000 ; 6$ : 13-17.
\end{abstract}

Key words : QT dispersion, Diabetic ulcer, Diabetic neuropathy, Arteriosclerosis

\section{Introduction}

Patients with non-insulin-dependent diabetes mellitus (NIDDM) are at great risk for foot ulceration. One reason is that arteriosclerosis of the foot arteries (arteriosclerosis obliterance) is more common in diabetic patients than in non-diabetic subjects (1). Poor blood supply (ischemia) due to arteriosclerosis is the major cause of foot ulcer, which is referred to as ischemic ulcer (2). Since foot artery disease is a manifestation of systemic artery sclerosis, it is often associated with coronary artery disease (3, 4). Patients with ischemic ulcer are therefore at high risk for myocardial infarction and sudden cardiac death.

Another reason for the increased incidence of foot ulcer in NIDDM is the frequent complication by diabetic polyneuropathy which causes peripheral sensory distur-

Address for correspondence: Eiichi Imano, M.D., Department of Gastroenterology and Metabolic Disease, Osaka Prefectural General Hospital, 3-1-56 Mandai-higashi, Sumiyoshi-ku, Osaka 558-8558, Japan.

Received September 16, 1999.

Accepted for publication may 31, 1999 bance and autonomic neuropathy that reduces sweating and toe blood pressure (5). Patients are often unaware of minor trauma and therefore foot ulcers develop easily. This type of lesion is referred to as neuropathic ulcer (2). Patients with neuropathic ulcers are prone to have cardiovascular autonomic neuropathy, e.g. prolongation of QT interval, possibly leading to life-threatening ventricular arrhythmias (6).

QT dispersion is a fairly new and simple variable currently under investigation in relation to cardiac disease $(7,8)$. It is obtained by calculating the QT interval for all 12 electrocardiographic leads and then subtracting the shortest interval from the longest interval. QT dispersion has been proposed to represent the inhomogeneity of ventricular repolarization $(9,10)$. Its increase has been reported in patients with myocardial ischemia (11) and ventricular arrhythmia $(9,12)$, and it is believed to have a predictive value for the risk of ventricular arrhythmias.

Because diabetic patients with ischemic or neuropathic ulcer are at a high risk for sudden cardiac death $(3,4,6)$, we hypothesized that these patients would show increased QT dispersion. 


\section{Subjects and Methods}

\section{Study groups}

We recruited 75 patients with NIDDM from our diabetic clinic. They were classified into four groups. None of them had any obvious cardiac diseases except those in the $\mathrm{MI}$ group and 1 patient with angina pectoris in the IU group. The absence of obvious cardiac disease was based on the fact that they had displayed no symptoms of coronary artery disease, no previous history of ischemic cardiac events and no electrocardiographic signs of ventricular hypertrophy or induced ischemia with Master's two-step test. Patients with electrolyte imbalance or overt proteinuria were not included.

(1) The NU group consisted of 15 patients who had neuropathic foot ulcer (ulcer lesions associated with paresthesia compatible with diabetic sensory-dominant peripheral neuropathy. Other possible etiologies that cause similar symptoms such as hereditary and toxic neuropathies had been excluded.) Arteriosclerotic foot disease was excluded since the resting ankle-brachial pressure index $(\mathrm{ABI})$ was more than 1.0.

(2) The IU group consisted of 20 patients with ischemic foot ulcer (ulcer lesions associated with arteriosclerosis). The diagnostic criterion was an $\mathrm{ABI}$ less than 0.8. Arteriosclerosis in 17 patients was confirmed by angiography. One patient with angina pectoris had undergone percutaneous transluminal coronary angioplasty. Concomitant diabetic peripheral neuropathy, based on the symptom of dysesthesia and decreased vibratory sense, was observed in 14 patients.

(3) The MI group consisted of 20 patients with a past history of myocardial infarction (4 to 24 months prior to the study). The patients received medication including nitrate derivatives (17 patients), beta-adrenergic blocking agents (12 patients), angiotensin-converting enzyme inhibitors (7 patients) and diuretic drugs (3 patients). However, there were no patients taking antiarrhythmic drugs or probucol which could affect the QT interval. Diabetic peripheral neuropathy was seen in 4 patients.

(4) The DC (diabetic control) group consisted of 20 patients without any signs of diabetic microangiopathies (normal findings in eye grounds, albumin excretion rate less than $20 \mu \mathrm{g} / \mathrm{min}$ )

As a control group, 15 inpatients with chronic hepatitis and normal glucose tolerance without obvious cardiac diseases were also studied (NC group).

\section{Patient characteristics}

Height and weight were recorded and body mass index (BMI) was calculated. We assessed the degree of cigarette smoking by pack-years of smoking history (past and current). Supine blood pressure was recorded with a sphygmomanometer after 10 minutes of rest. The heart rate response during deep breathing over 1 minute was recorded and beat-to-beat variation between expiration and inspiration was calculated.

Blood was withdrawn after overnight fasting for analysis of total cholesterol and high-density lipoprotein (HDL) cholesterol, triglyceride and glycated hemoglobin. The levels of glycated hemoglobin were measured with highperformance liquid chromatography with a reference value of $3.5-6.5 \%$. Total cholesterol and triglyceride levels were enzymatically determined (cholesterol oxidase and lipoprotein lipase methods). High-density lipoprotein (HDL) cholesterol was measured with Polyethylene Glycol-modified Enzymes and Sulfated alfa-Cyclodextrin. Urine was collected for 24 hours and the albumin excretion rate (AER, $\mu \mathrm{g} / \mathrm{min}$ ) was quantitated by radioimmunoassay. All subjects were confirmed to be free of urinary tract infections.

\section{Electrocardiographic analysis}

The electrocardiogram $(25 \mathrm{~mm} / \mathrm{sec}$ speed) from 12 surface leads was used to determine the QT interval from the onset of the QRS complex to the end of the $T$ wave (return to the $\mathrm{T} / \mathrm{P}$ baseline). If $\mathrm{U}$ waves were present, the QT interval was measured to the nadir of the curve between the $T$ and $U$ waves. If the end of the $T$ wave could not be reliably determined, QT measurements were not taken and these leads were excluded from analysis. A single observer, unaware of the patients' profiles, measured the QT intervals for as many leads as possible (minimum 9 and mean 10.1 leads). Three consecutive cycles were measured for each lead and averaged. QT intervals were corrected with Bazett's formula (QTc $=Q T$ / $R^{1 / 2}$ ) (13). Since Bazett's formula overcorrects for a high heart rate, electrocardiograms with a moderate (70-90/ min.) heart rate and normal sinus rhythm were selected. QTC dispersion, defined as the difference between maximum and minimum QTc in all measurable leads, was calculated.

\section{Statistical analysis}

Data are given as mean $\pm S D$ values and differences among the five groups were analyzed by one-way ANOVA. As for diabetic duration, degree of cigarette smoking, AER, and beat-to-beat variation, data are presented as median and interquartiles and analyzed by Kruskal-Wallis test. Post hoc group comparisons were made with Scheffe's $F$ test. A value of $P<0.05$ was considered significant.

\section{Results}

\section{Patient characteristics}

The clinical characteristics of the five study groups are summarized in Table 1. There were no significant differences in age and BMI among the groups. Diabetic duration in the NU and IU groups was longer than that in the DC group $(P<0.05)$. Pack-years (degree of smoking) in the IU group was significantly higher than that in the DC 
Table 1. Clinical characteristics of the five groups studied

\begin{tabular}{|c|c|c|c|c|c|}
\hline & \multirow{2}{*}{ NC } & \multicolumn{4}{|c|}{ NIDDM } \\
\hline & & DC & $\mathrm{NU}$ & IU & $\mathrm{Ml}$ \\
\hline Age $(y r)$ & $62 \pm 12$ & $58 \pm 12$ & $62 \pm 12$ & $64 \pm 8$ & $60 \pm 8$ \\
\hline $\mathrm{BMI}\left(\mathrm{kg} / \mathrm{m}^{-2}\right)$ & $24 \pm 4$ & $25 \pm 4$ & $21 \pm 4$ & $22 \pm 4$ & $23 \pm 3$ \\
\hline Diabetic duration \#(yr) & - & $3(0.6,5)$ & $17(7,20)^{+}$ & $16(6,20)^{+}$ & $5(3,7)$ \\
\hline Treatment: D/OHA/I & - & $13 / 6 / 1$ & $4 / 4 / 7$ & $7 / 9 / 4$ & $7 / 11 / 2$ \\
\hline Pack-years\# & $22(20,56)$ & $12(5,47)$ & $18(7,49)$ & $45(20,54)^{+}$ & $30(12,42)$ \\
\hline $\mathrm{HbA}_{1 \mathrm{c}}(\%)$ & $5.1 \pm 0.7$ & $9.0 \pm 2.8^{*}$ & $9.1 \pm 1.8^{*}$ & $8.1 \pm 1.6^{*}$ & $8.6 \pm 2.6^{*}$ \\
\hline $\mathrm{TC}(\mathrm{mg} / \mathrm{dl})$ & $196 \pm 40$ & $195 \pm 32$ & $170 \pm 42$ & $182 \pm 39$ & $192 \pm 38$ \\
\hline $\mathrm{TG}(\mathrm{mg} / \mathrm{dl})$ & $153 \pm 53$ & $148 \pm 36$ & $136 \pm 33$ & $130 \pm 52$ & $165 \pm 56$ \\
\hline $\mathrm{HDL}-\mathrm{C}(\mathrm{mg} / \mathrm{dl})$ & $63 \pm 21$ & $45 \pm 18$ & $56 \pm 20$ & $41 \pm 13$ & $43 \pm 12$ \\
\hline $\operatorname{AER}^{\#}(\mu \mathrm{g} / \mathrm{min})$ & N.A. & $7.9(3.8,13.1)$ & $10.4(6.8,14.6)$ & $79.6(20.9,98.7)^{+}$ & $32.1(10.7,47.6)^{+}$ \\
\hline $\mathrm{B} / \mathrm{B}$ variation ${ }^{*}($ beats $/ \mathrm{min})$ & $12.0(8.0,14.0)$ & $11.4(8.8,14.4)$ & $6.2(4.2,7.3)^{+*}$ & $6.4(4.0,7.8)^{+*}$ & $7.4(6.1,13.8)^{+*}$ \\
\hline Supine SBP $(\mathrm{mmHg})$ & $124 \pm 18$ & $123 \pm 12$ & $130 \pm 22$ & $139 \pm 23$ & $124 \pm 19$ \\
\hline Supine DBP (mmHg) & $76 \pm 10$ & $74 \pm 19$ & $75 \pm 9$ & $75 \pm 15$ & $75 \pm 11$ \\
\hline
\end{tabular}

\#: Median values, 25th and 75th percentiles.

${ }^{+}$: Signifies a difference from the DC group.

*: Signifies a difference from the NC group.

$\mathrm{NIDDM}=$ non-insulin-dependent diabetes mellitus ; $\mathrm{NC}=$ non-diabetic control subjects ; $\mathrm{DC}=$ diabetic patients without any angiopathy (diabetic control subjects) ; $\mathrm{NU}=$ patients with neuropathic ulcer ; IU = patients with ischemic ulcer; $\mathrm{MI}=$ patients with previous myocardial infarction; $B M I=$ body mass index; $D=\operatorname{diet} ; O H A=$ oral hypoglycemic agents ; $I=$ insulin ; $\mathrm{HbA}_{1 \mathrm{C}}=$ glycated hemoglobin ; $\mathrm{TC}=$ total cholesterol ; $\mathrm{TG}=$ triglyceride ; $\mathrm{HDL}-\mathrm{C}=$ high-density lipoprotein cholesterol ; $\mathrm{AER}=$ albumin excretion rate ; $\mathrm{B} / \mathrm{B}=$ beat to beat ; $\mathrm{SBP}=$ systolic blood pressure ; $\mathrm{DBP}=$ diastolic blood pressure; N.A. $=$ not available.

group.

Glycated hemoglobin levels in four NIDDM groups were significantly higher than that in the NC group. Serum lipid levels (total cholesterol, HDL-cholesterol and triglyceride) did not differ among the groups. AERs in the IU and MI groups were significantly higher than that in the DC group. In the IU group, 14 of 20 patients had microalbuminuria (AER; $20-200 \mu \mathrm{g} / \mathrm{min}$ ). Beat-to-beat variations in the NU, IU and $\mathrm{MI}$ groups were significantly smaller than in the NC or DC groups $(P<0.05)$. Supine systolic or diastolic blood pressures did not differ among the groups.

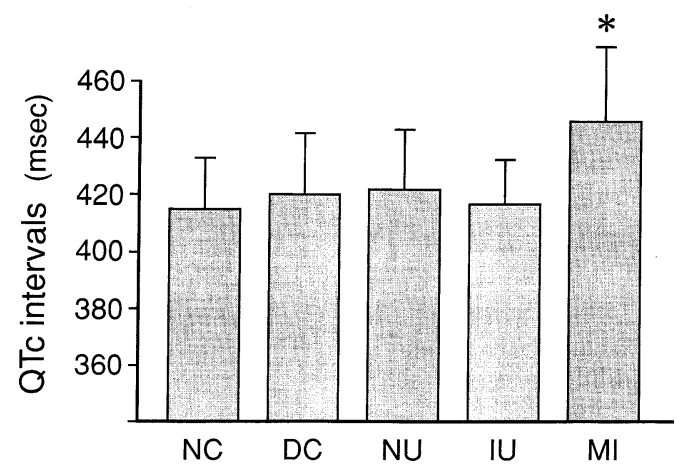

Fig. 1-A. QTC intervals in non-diabetic controls (NC), and diabetic patients without microangiopathy (DC), with neuropathic ulcer (NU), with ischemic ulcer (IU) or previous history of myocardial infarction (MI). $\quad+: P<0.05$ vs. DC group. * : $P<0.05$ vs. NC group.

\section{QT dispersion analysis}

The QTc interval in the MI group was significantly higher $(446 \pm 27 \mathrm{msec}, P<0.05)$ than that in the NC $(415 \pm$ $16 \mathrm{msec})$ or DC group $(420 \pm 22 \mathrm{msec})$, but not in the NU $(422 \pm 22 \mathrm{msec})$ or IU group ( $416 \pm 15 \mathrm{msec}$ ) (Fig. 1-A). QT dispersions in the NU group $(52 \pm 10 \mathrm{msec}, P<0.05)$ and the IU group $(54 \pm 15 \mathrm{msec}, P<0.05)$ as well as the $\mathrm{MI}$ group $(60 \pm 21 \mathrm{msec}, P<0.05)$ were significantly higher than those in the $\mathrm{NC}(36 \pm 12 \mathrm{msec})$ or DC group $(39 \pm 8$ msec) (Fig. 1-B).

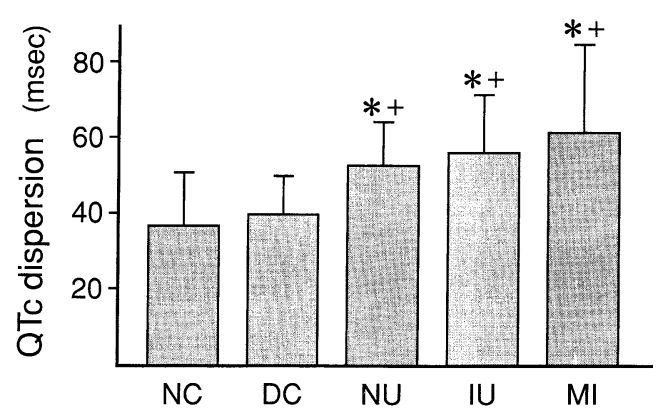

Fig. 1-B. QT dispersions in non-diabetic controls (NC), and diabetic patients without microangiopathy (DC), with neuropathic ulcer (NU), with ischemic ulcer (IU) or previous history of myocardial infarction (MI). $+: P<0.05$ vs. DC group. * : $P<0.05$ vs. NC group. 


\section{Discussion}

Foot lesion is a serious health problem that impairs the patient's quality of life. Since diabetic neuropathy and arteriosclerosis are important etiologic factors in diabetic ulcer, foot ulcer can be classified as a neuropathic or ischemic ulcer (2). About $40-60 \%$ of patients with ischemic ulcer have been shown to have coronary artery disease $(3,4)$. Furthermore, patients with neuropathic ulcer are prone to have cardiovascular autonomic neuropathy, e.g. prolongation of QT interval, possibly leading to life-threatening ventricular arrhythmias (6). Patients with diabetic foot ulcer are therefore at a high risk for sudden cardiac death. Although many of the patients are asymptomatic, it is worthwhile to identify patients at high risk for cardiac events so that they can receive appropriate cardiac management.

QT dispersion is a fairly new and simple measure that reflects inhomogeneous ventricular repolarization (7-10), i.e. spatial dispersion of electrical refractoriness in the ventricular muscle. Increased QT dispersion has been reported in patients with myocardial ischemia (11) and ventricular arrhythmia $(9,12)$, and it is considered useful for identifying patients at increased risk of arrhythmic events. In addition to that, diabetic patients with autonomic neuropathy have been shown to have increased QT dispersion (13-15). We therefore hypothesized that diabetic patients with foot ulcers would also have increased QT dispersion.

QT dispersions in the IU group as well as the MI group were significantly higher than those in the NC or DC group. Ischemic ulcer is a partial manifestation of systemic arteriosclerosis and it is frequently associated with coronary artery disease $(3,4)$. Although patients in the IU group did not show ischemic changes on electrocardiograms with Master two-step test, taking low sensitivity of the test into account, the patients in the IU group may have asymptomatic coronary artery disease. Indeed, 14 of 20 patients had microalbuminuria which is considered a strong predictor of cardiovascular disease (16). We assume that complicated atherosclerotic heart disease may contribute to increased QT dispersions in the IU group. Darbar et al. found that there was a strong link between QT dispersion and cardiac death in patients with arteriosclerotic foot artery disease (17). They revealed that patients with diffuse coronary artery disease had the greatest QT dispersion among those with coronary artery disease (1-3 vessels affected). We thought it would be valuable to investigate QT dispersion in patients with diabetic cardiomyopathy, since diffuse endothelial proliferation of small coronary arteries has been reported in such patients (18).

Another thing we should consider is the complication of cardiac autonomic dysfunction. QT dispersion in the NU group was also increased. Decreased parasympathetic nerve function reflected by a reduced beat-to-beat varia- tion was shown in the IU as well as the NU group. Previous studies suggested that decreased parasympathetic tone increases QT dispersion (19). The increased QT dispersion in the NU group may be due to decreased cardiac parasympathetic activity. We also speculate that the complication of cardiac autonomic dysfunction is relevant to the increased QT dispersion in the IU group. Further study will provide evidence to confirm or deny this assumption. For example, MIBG scintigram would estimate the cardiac sympathetic dysfunction and thallium scintigram would show the degree of myocardial ischemia.

Diabetic patients with either neuropathic or ischemic ulcer are at a high risk for cardiac death. This may be further supported by our finding that QT dispersion was increased in patients with neuropathic or ischemic ulcer. Although the precise cause of increased QT dispersion cannot be determined, cardiac ischemia or autonomic dysfunction, it is quite possible that the patients share the common characteristics of inhomogeneous ventricular repolarization leading to sudden cardiac death. Prolongation of the QT interval has been reported in diabetic patients with autonomic neuropathy (20). However, in this study, the QTC interval was increased only in the MI group and not in the NU or IU group. QT dispersion could be a more sensitive predictor for ventricular arrhythmias than the QT interval in a single lead. However, since the causal relation among diabetic ulcer, QT dispersion and cardiac sudden death has not been clearly elucidated, a prospective study is needed as to the incidence of sudden cardiac death and the coronary heart disease in the patients.

In conclusion, we have revealed that QT dispersion is increased in patients with diabetic ulcer through atherosclerosis or neurological disorder. Although the precise mechanism of this increase is unknown, QT dispersion may predict the complication of autonomic dysfunction or coronary artery disease and even the potential risk for sudden cardiac death.

\section{References}

(1) Walters DP, Gatling W, Mullee MA, and Hill RD: The prevalence, detection and epidemiological correlates of peripheral vascular disease: a comparison of diabetic and non-diabetic subjects in an English community. Diabetic Med, 9: 710-715, 1992

(2) Shaw JE and Boulton AJ: The pathogenesis of diabetic foot problems. An overview. Diabetes, 46 (Suppl 2) : S58-S61, 1997

(3) Hertzer NR, Beven EG, and Young JR: Coronary artery disease in peripheral vascular patients. Ann Sug, 199: 222-233, 1984

(4) Gajraj H and Jamieson CW : Coronary artery disease in patients with peripheral vascular disease. $\mathrm{Br} J$ Surg, 81 : 333-342, 1994

( 5 ) Uccioli L, Monticone G, Durola L, Russo F, Mormile F, 
Mennuni G, and Menzinger G : Autonomic neuropathy influences great toe blood pressure. Diabetes Care, 17 : 284-287, 1994

(6) Kahn JK, Sisson JC, and Vinik AI : QT interval prolongation and sudden cardiac death in diabetic autonomic neuropathy. J Clin Endocrinol Metab, 64: 751-754, 1987

(7) Cowan JC, Yusoff K, Moor M, and Campbell RWF: Importance of lead selection in QT interval measurement. Am J Cardiol, 61: 83-87, 1988

(8) Higham PD and Campbell RWF : QT dispersion. $\mathrm{Br}$ Heart J, 71: 508-509, 1994

(9) Day CP, McComb JM, and Campbell RWF : QT dispersion: an indication of arrhythmia risk in patients with long QT intervals. Br Heart J, 63: 342-344, 1990

(10) Statters DJ, Malik M, Ward DE, and Camm AJ : QT dispersion : problems of methodology and clinical significance. Cardiovasc Electrophysiol, 5 : 672-685, 1994

(11) Higham PD, Furniss SS, and Campbell RW : QT dispersion and component of the QT interval in ischaemia and infarction. Br Heart J, 73: 32-36, 1995

(12) Linker NJ, Colonna P, Kekwick CA, Till J, Camm J, and Ward DE : Assessment of QT dispersion in symptomatic patients with congenital long QT syndrome. Am J Cardiol, 69: 634-638, 1992

(13) Kirvela M, Yli-Hankala A, and Lindgren L: QT dispersion and autonomic function in diabetic and non-diabetic patients with renal failure. $\mathrm{Br} J$ Anaesth, 73: 801-804, 1994
(14) Wei K, Dorian P, Newman D, and Langer A : Association between QT dispersion and autonomic dysfunction in patients with diabetes mellitus. J Am Coll Cardiol, 26 : 859-863, 1995

(15) Shimabukuro $M$, Chibana $T$, Yoshida $H$, Nagamine $F$, Komiya I, and Takasu N : Increased QT dispersion and cardiac adrenergic dysinnervation in diabetic patients with autonomic neuropathy. Am J Cardiol, 78: 10571059, 1996

(16) Schmitz $A$ and Vaeth $M$ : Microalbuminuria : a major risk factor in non-insulin-dependent diabetes. A 10-year follow-up study of 503 patients. Diabet Med, 5 : 126134, 1988

(17) Darbar D, Luck J, Davidson N, Pringle T, Main G, McNeill $G$, and Struthes AD: Sensitivity and specificity of QTC dispersion for identification of risk of cardiac death in patients with peripheral vascular disease. BMJ, 312 : 874-878, 1996

(18) Hamby RI, Zoneraich S, and Sherman S: Diabetic cardiomyopathy. JAMA, 229: 1749-1754, 1974

(19) Ishida S, Nakagawa M, Fujino T, Yonemochi H, Saikawa $T$, and Ito $M$ : Circadian variation of $Q T$ dispersion : correlation with heart rate variability. J Electrocardiol, 30 : 205-210, 1997

(20) Conin JM, Kadroske MM, Schmaltz S, Bastyr EJ, and Vinik AL: Corrected QT interval prolongation as diagnostic tool for assessment of cardiac autonomic neuropathy in diabetes mellitus. Diabetes Care, 13: 68-71, 1990 